\title{
Two-level finite element method with a stabilizing subgrid for the incompressible Navier-Stokes equations
}

\author{
A. I. Nesliturk ${ }^{1,2, *, \dagger}$, S. H. Aydın² and M. Tezer-Sezgin ${ }^{2,3}$ \\ ${ }^{1}$ Department of Mathematics, Izmir Institute of Technology, 35430 Izmir, Turkey \\ ${ }^{2}$ Institute of Applied Mathematics, Middle East Technical University, 06531 Ankara, Turkey \\ ${ }^{3}$ Department of Mathematics, Middle East Technical University, 06531 Ankara, Turkey
}

\begin{abstract}
SUMMARY
We consider the Galerkin finite element method for the incompressible Navier-Stokes equations in two dimensions. The domain is discretized into a set of regular triangular elements and the finite-dimensional spaces employed consist of piecewise continuous linear interpolants enriched with the residual-free bubble functions. To find the bubble part of the solution, a two-level finite element method with a stabilizing subgrid of a single node is described, and its application to the Navier-Stokes equation is displayed. Numerical approximations employing the proposed algorithm are presented for three benchmark problems. The results show that the proper choice of the subgrid node is crucial in obtaining stable and accurate numerical approximations consistent with the physical configuration of the problem at a cheap computational cost. Copyright (C) 2008 John Wiley \& Sons, Ltd.
\end{abstract}

Received 22 May 2007; Revised 26 November 2007; Accepted 18 December 2007

KEY WORDS: stabilizing subgrid; Navier-Stokes equations; two-level finite element method

\section{INTRODUCTION}

Applications of the Galerkin finite element method to incompressible Navier-Stokes equations in velocity-pressure form were carried out in the early 1970s. It was soon recognized that the use of equal-order interpolations for both velocity and pressure variables, which is the most desirable choice from the implementation point of view, generates numerical approximations that is inconsistent with the physical configuration of the problem $[1,2]$. The difficulty is two-fold. In the first layer, the finite element formulation of the problem is of mixed form and the appropriate pair of the function spaces satisfying the Babuška-Brezzi condition must be employed [3-5].

\footnotetext{
${ }^{*}$ Correspondence to: A. I. Nesliturk, Department of Mathematics, Izmir Institute of Technology, 35430 Izmir, Turkey.

†E-mail: alinesliturk@iyte.edu.tr

Contract/grant sponsor: The Scientific and Technical Research Council of Turkey (TUBITAK); contract/grant number: $105 \mathrm{~T} 091$
}

Copyright (C) 2008 John Wiley \& Sons, Ltd. 
However, the problem may still exhibit numerical instabilities at high Reynolds numbers even when appropriate pair of approximation spaces is employed. A considerable amount of scientific work has been devoted to developing numerical algorithms that are able to cope with both of these problems simultaneously.

One of the most popular of such numerical methods is referred to as the streamline upwind Petrov-Galerkin (SUPG) method [6]. The finite element methods of the SUPG type reduces the oscillations in the standard Galerkin method of piecewise linears and achieves stability by adding mesh-dependent perturbation terms to the formulation. These terms enhance the coercivity of the formulation by acting like artificial diffusion in the direction of streamlines and enables the usage of the velocity-pressure pairs that are known to produce approximations not consistent with the exact solution of the problem [7]. However, the amount of perturbation or the value of the stability parameters that should be chosen by the user is not known a priori and needs to be adjusted by means of error analysis and/or experiments.

Later it has been shown that the SUPG-type stabilized methods for the equations modeling the flow problems can be derived by adding the bubble functions to the velocity space in the standard Galerkin finite element formulation and then eliminating the bubbles by using the static condensation approach [8-14]. In this approach the optimal choice of the stabilization parameter in the SUPG method was simply translated into the problem of the optimal choice of the bubble space. Therefore, the bubble functions should be chosen appropriately to cope with the difficulties arising in the numerical simulation of the equations. In that context, the residual-free bubble (RFB) functions whose description is based on a local boundary value problem related to the strong form of the equation in each element were introduced in [9]. The numerical methods employing the RFB functions were investigated in different element configurations mostly for advection-dominated flows. It can be verified that the RFB method enhances the stability of the discrete problem and satisfies a priori error estimates similar to the ones for the SUPG method [15-20].

Since the RFB method is based on the classical solution of a local problem of advection-diffusion type, finding its exact solution is usually difficult as much as the original system of differential equations. Therefore, an accurate numerical algorithm to obtain the approximate solution of the bubble problem inside each element has to be designed. This can be done by the two-level finite element method (TLFEM). The TLFEM was first introduced by Franca and Macedo in [21] for the Helmholtz equation. It was later extended to the advection-diffusion equation in Franca et al. [16] and to the incompressible Navier-Stokes equations in [22]. TLFEM consists of two parts: On the one at the global mesh level, the problem will be in the Galerkin framework, whereas the bubble part of the solution is still unknown. Before solving the problem on the global mesh, we have to compute the bubble functions, which is accomplished in the second part of the method. To do this, we set another layer of mesh (subgrid) inside each element on which we calculate the approximate solution of bubble functions by using a nonstandard finite element method. Then these approximations are used instead of the exact bubble functions in the global mesh level of the formulation. However, the implementation of the TLFEM can be expensive from the computational point of view, and a cheap efficient algorithm that generates qualitatively the same bubble functions as the TLFEM thus is sought.

Recently, some numerical algorithms were proposed in the context of advection-diffusion equation to provide a cheap approximate solution to the bubble problem with the use of a subgrid consisting of very few nodes [23,24]. The method can be viewed as a variant of the TLFEM where the subgrid consisted of a single internal node plus three vertices induced by the global mesh, per element. The internal node is taken over one of the medians of the triangle and the 
precise location of the subgrid node is determined so that a residual value becomes minimum in the sense of $L_{1}$. With the use of a suitably chosen internal subgrid node, it can be proven that the resulting numerical method, called stabilizing subgrid method (SSM), has qualitatively similar stability features of the classical stabilized methods and satisfies the same a priori error estimates as the SUPG or the RFB methods. However, these results hold only if the advection field is piecewise constant inside each element.

In this work, we apply the SSM to the approximation of the incompressible Navier-Stokes equations on a triangular discretization of the domain. We employ the space of continuous piecewise linears plus bubbles for the velocity variable and the continuous piecewise linears for the pressure variable in a framework of the mixed finite element method. The nonlinearity emanating from the nature of the equation is treated through an iteration. We assume that the advection field is uniform inside each element by taking the average value of the velocity variable at the vertices of the triangle. The resulting numerical method approximates well and proves good stability features. It is further computationally cheap and able to adapt itself between different flow regimes.

We organize the paper as follows: In Section 2, we describe the RFB formulation of the NavierStokes equations and the part of the TLFEM that consists of the contribution of the RFB functions to the global problem. The second part of TLFEM that consists of approximating to the exact bubble functions using the SSM is presented in Section 3. Implementation issues related to the subgrid will also be discussed in this section. We illustrate the potential of the method in Section 4 by presenting some numerical experiments obtained in different problem configurations. Conclusions are drawn in Section 5.

\section{THE RFB METHOD FOR THE INCOMPRESSIBLE NAVIER-STOKES EQUATIONS}

\subsection{Steady-state Navier-Stokes Equations}

The steady-state incompressible Navier-Stokes equations in an open-bounded domain $\Omega \subset \mathbb{R}^{2}$ with the boundary $\partial \Omega$ are given by

$$
\begin{aligned}
(\nabla \mathbf{u}) \mathbf{u}-\varepsilon \Delta \mathbf{u}+\nabla p=\mathbf{f} & & \text { in } \Omega \\
\nabla \cdot \mathbf{u}=0 & & \text { in } \Omega \\
\mathbf{u}=0 & & \text { on } \partial \Omega
\end{aligned}
$$

where $\mathbf{u}$ is the unknown velocity field, $p$ is the unknown scalar pressure function, $\mathbf{f}$ is a given source function and $\varepsilon$ is the viscosity parameter. The weak formulation of problem (1) is obtained by employing the pair of function spaces $V=\left(H_{0}^{1}(\Omega)\right)^{2}$ and $P=C^{0}(\bar{\Omega}) \cap L_{0}^{2}(\Omega)$, and it reads: Find $\mathbf{u} \in V, p \in P$ such that

$$
B(\mathbf{u} ; \mathbf{u}, p ; \mathbf{v}, q)=F(\mathbf{v}, q) \quad \text { for all } \mathbf{v} \in V, \quad q \in P
$$

where the forms are given by

$$
\begin{gathered}
B(\mathbf{w} ; \mathbf{u}, p ; \mathbf{v}, q)=((\nabla \mathbf{u}) \mathbf{w}, \mathbf{v})+\varepsilon(\nabla \mathbf{u}, \nabla \mathbf{v})-(\nabla \cdot \mathbf{v}, p)+(\nabla \cdot \mathbf{u}, q) \\
F(\mathbf{v}, q)=(\mathbf{f}, \mathbf{v})
\end{gathered}
$$

A mixed finite element approximation of problem (2) is determined by the choice of finitedimensional subspaces $V_{h} \subset V, P_{1} \subset P$ defined on a family of discretizations $T_{h}$ of the domain $\Omega$. 
We assume that $T_{h}$ is made of triangles $\{K\}$ and that $T_{h}$ is regular as defined in [25]. Denoting the space of piecewise linear functions on a typical element $K$ by $P_{1}(K)$, we choose the following finite-dimensional subspaces on $T_{h}$ before we specify a finite element method:

$$
\begin{gathered}
V_{h}=V_{1} \oplus \bigcup_{K \in T_{h}}(B(K))^{2}=\left\{\mathbf{v} \in\left(H_{0}^{1}(\Omega)\right)^{2}|\mathbf{v}|_{K} \in\left(P_{1}(K) \oplus B(K)\right)^{2}, K \in T_{h}\right\} \\
V_{1}=\left\{\mathbf{v} \in\left(H_{0}^{1}(\Omega)\right)^{2}|\mathbf{v}|_{K} \in P_{1}(K)^{2}, K \in T_{h}\right\} \\
P_{1}=\left\{p \in C^{0}(\bar{\Omega}) \cap L_{0}^{2}(\Omega)|p|_{K} \in P_{1}(K), K \in T_{h}\right\}
\end{gathered}
$$

where $B(K)$ is the space of RFB functions, which we define later. The standard Galerkin finite element method is based on employing the same function space for both test and trial spaces and it is equivalent to finding the pair $\left\{\mathbf{u}_{h}=\mathbf{u}_{1}+\mathbf{u}_{b}, p_{1}\right\}$ from $V_{h} \times P_{1}$ such that

$$
B\left(\mathbf{u}_{h} ; \mathbf{u}_{h}, p_{1} ; \mathbf{v}_{h}, q_{1}\right)=\left(\mathbf{f}, \mathbf{v}_{h}\right) \quad \forall\left\{\mathbf{v}_{h}, q_{1}\right\} \in V_{h} \times P_{1}
$$

where

$$
\begin{aligned}
B\left(\mathbf{w}_{h} ; \mathbf{u}_{h}, p_{1} ; \mathbf{v}_{h}, q_{1}\right)= & \left(\left(\nabla \mathbf{u}_{h}\right) \mathbf{w}_{h}, \mathbf{v}_{h}\right)+\varepsilon\left(\nabla \mathbf{u}_{h}, \nabla \mathbf{v}_{h}\right) \\
& -\left(\nabla \cdot \mathbf{v}_{h}, p_{1}\right)+\left(\nabla \cdot \mathbf{u}_{h}, q_{1}\right)
\end{aligned}
$$

Problem (3) is nonlinear due to the presence of the advection term, which will be resolved by employing an iteration process: Let us decompose the approximate solutions $\mathbf{u}_{h}$ and $p_{1}$ as

$$
\begin{aligned}
& \mathbf{u}_{h}^{n+1}=\mathbf{u}_{h}^{n}+\hat{\mathbf{u}}_{h} \\
& p_{1}^{n+1}=p_{1}^{n}+\hat{p}_{1}
\end{aligned}
$$

where $\mathbf{u}_{h}^{n+1}$ and $p_{1}^{n+1}$ are the approximations at the current iteration step, $\mathbf{u}_{h}^{n}$ and $p_{1}^{n}$ are the approximations at the previous iteration step, and $\hat{\mathbf{u}}_{h}$ and $\hat{p}_{1}$ are the corrections to the approximations at the previous iteration step. We linearize problem (3) by replacing the advection term $\left(\left(\nabla \mathbf{u}_{h}\right) \mathbf{u}_{h}, \mathbf{v}_{h}\right)$ with $\left(\left(\nabla \mathbf{u}_{h}^{n+1}\right) \mathbf{u}_{1}^{n}, \mathbf{v}_{h}\right)$. Now if we choose the space of bubble functions such that a typical bubble function $\mathbf{u}_{b}$ in $B(K)$ is zero outside a fixed element $K \in T_{h}$ and satisfy the momentum equation inside $K$; that is

$$
\begin{aligned}
\nabla\left(\mathbf{u}_{1}^{n+1}+\mathbf{u}_{b}^{n+1}\right) \mathbf{u}_{1}^{n}-\varepsilon \triangle\left(\mathbf{u}_{1}^{n+1}+\mathbf{u}_{b}^{n+1}\right)+\nabla p_{1}^{n+1}=\mathbf{f} & \text { in } K \\
\mathbf{u}_{b}^{n+1}=0 & \text { on } \partial K
\end{aligned}
$$

then this special choice of bubble functions in (6), the so-called RFB, enables us to employ the static condensation procedure, from which we obtain the Galerkin formulation using piecewise linears for problem (1) modified by the RFB: Find $\left\{\mathbf{u}_{h}^{n+1}, p_{1}^{n+1}\right\} \in V_{h} \times P_{1}$ such that

$$
B\left(\mathbf{u}_{1}^{n} ; \mathbf{u}_{h}^{n+1}, p_{1}^{n+1} ; \mathbf{v}_{1}, q_{1}\right)=\left(\mathbf{f}, \mathbf{v}_{1}\right) \quad \forall\left\{\mathbf{v}_{1}, q_{1}\right\} \in V_{1} \times P_{1}
$$

where

$$
\begin{aligned}
B\left(\mathbf{u}_{1}^{n} ; \mathbf{u}_{h}^{n+1}, p_{1}^{n+1} ; \mathbf{v}_{1}, q_{1}\right)= & \left(\left(\nabla \mathbf{u}_{h}^{n+1}\right) \mathbf{u}_{1}^{n}, \mathbf{v}_{1}\right)+\varepsilon\left(\nabla \mathbf{u}_{h}^{n+1}, \nabla \mathbf{v}_{1}\right) \\
& -\left(\nabla \cdot \mathbf{v}_{1}, p_{1}^{n+1}\right)+\left(\nabla \cdot \mathbf{u}_{h}^{n+1}, q_{1}\right)
\end{aligned}
$$


A rearrangement of terms in (7) reveals the explicit contribution of the RFB functions to the standard Galerkin formulation of the problem. The problem now reads: Find $\left\{\mathbf{u}_{h}^{n+1}, p_{1}^{n+1}\right\} \in V_{h} \times P_{1}$ such that

$$
B\left(\mathbf{u}_{1}^{n} ; \mathbf{u}_{1}^{n+1}, p_{1}^{n+1} ; \mathbf{v}_{1}, q_{1}\right)+\left(\left(\nabla \mathbf{u}_{b}^{n+1}\right) \mathbf{u}_{1}^{n}, \mathbf{v}_{1}\right)+\varepsilon\left(\nabla \mathbf{u}_{b}^{n+1}, \nabla \mathbf{v}_{1}\right)+\left(\nabla \cdot \mathbf{u}_{b}^{n+1}, q_{1}\right)=\left(\mathbf{f}, \mathbf{v}_{1}\right)
$$

holds for all $\left\{\mathbf{v}_{1}, q_{1}\right\} \in V_{1} \times P_{1}$, where the first term comes from the standard Galerkin formulation of the problem with piecewise linears only, which is known to be unstable. These additional terms make the numerical method recover the physical structure of the problem and, thus, are responsible for stability of the numerical method. Further, it can be proved that the elimination of the RFB functions in these additional terms leaves behind a formulation similar to a stabilized finite element method of the SUPG type [12]: Find $\left\{\mathbf{u}_{1}^{n+1}, p_{1}^{n+1}\right\} \in V_{1} \times P_{1}$ such that

$$
\begin{gathered}
B\left(\mathbf{u}_{1}^{n} ; \mathbf{u}_{1}^{n+1}, p_{1}^{n+1} ; \mathbf{v}_{1}, q_{1}\right)+\sum_{K \in T_{h}} \tau_{K} \int_{K}\left[\left(\nabla \mathbf{u}_{1}^{n+1}\right) \mathbf{u}_{1}^{n}+\nabla p_{1}^{n+1}-\mathbf{f}\right]\left[\left(\nabla \mathbf{v}_{1}\right) \mathbf{u}_{1}^{n}-\nabla q_{1}\right]=\left(\mathbf{f}, \mathbf{v}_{1}\right) \\
\forall\left\{\mathbf{v}_{1}, q_{1}\right\} \in V_{1} \times P_{1}
\end{gathered}
$$

where the stabilization parameter $\tau_{K}$ is explicitly given by

$$
\tau_{K}=\frac{1}{|K|} \int_{K} b_{K} \mathrm{~d} K
$$

and $b_{K}$ is the unique bubble function defined by the following boundary value problem in $K$ :

$$
\begin{aligned}
L b_{K}=-\varepsilon \triangle b_{K}+\mathbf{u}_{1}^{n} \cdot \nabla b_{K}=1 & \text { in } K \\
b_{K}=0 & \text { on } \partial K
\end{aligned}
$$

We note that Equation (9) and the $S U P G$ formulation of the Navier-Stokes equations have the identical structure except for the value of the stabilization parameter $\tau_{K}$. The value of the stabilization parameter $\tau_{K}$ is given in terms of the bubble function $b_{K}$ for which, finding its exact solution using (12) may not be an easy task in an arbitrary triangular domain. Therefore a cheap efficient approximation by $b_{K}$ that generates qualitatively the same behavior with the exact bubble function $b_{K}$ is required, and its algorithm is presented in Section 3.

\subsection{Unsteady Navier-Stokes equations}

The unsteady Navier-Stokes equations can be given by

$$
\begin{aligned}
\frac{\partial \mathbf{u}}{\partial t}+(\nabla \mathbf{u}) \mathbf{u}-\varepsilon \Delta \mathbf{u}+\nabla p & =\mathbf{f} & & \text { in } \Omega \times(0, T) \\
\nabla \cdot \mathbf{u} & =0 & & \text { in } \Omega \times(0, T) \\
\mathbf{u} & =\mathbf{g} & & \text { on } \partial \Omega \times(0, T) \\
\mathbf{u}(0, .) & =\mathbf{u}_{0} & & \text { in } \Omega \\
\int_{\Omega} p \mathrm{~d} \mathbf{x} & =0 & & \text { in } \Omega \times(0, T)
\end{aligned}
$$


where $\mathbf{u}$ is the velocity, $\mathbf{u}_{0}$ is the initial velocity, $p$ is the pressure, $\mathbf{f}$ represents the body force, $(0, T)$ is a given time interval, $\Omega \subset R^{2}$ is a bounded domain with the boundary $\partial \Omega$ and $\mathbf{g}$ is a given function defined on the boundary.

We approximate the time derivative terms by using the finite difference method as

$$
\mathbf{u}^{s+1}-\mathbf{u}^{s}=\Delta t\left[\alpha \dot{\mathbf{u}}^{s+1}+(1-\alpha) \dot{\mathbf{u}}^{s}\right]
$$

where $\dot{\mathbf{u}}=\partial \mathbf{u} / \partial t, \alpha$ is the relaxation parameter with $\alpha \in[0,1]$ and $\Delta t=t_{s+1}-t_{s}$, the length of the time interval in a typical slab $\left[t_{s}, t_{s+1}\right] \times \Omega$. For special values of $\alpha$, the method turns into one of the following well-known time-stepping procedures:

- $\alpha=1$ : Implicit type.

- $\alpha=\frac{1}{2}$ : Crank-Nicolson type.

- $\alpha=0$ : Explicit type.

The spatial discretization of the weak form of (13) leads to the following equation: Find $\left\{\mathbf{u}_{h}, p_{1}\right\}$ in $V_{h} \times P_{1}$ such that

$$
B\left(\mathbf{u}_{h} ; \mathbf{u}_{h}, p_{1} ; \mathbf{v}_{h}, q_{1}\right)=\left(\mathbf{f}, \mathbf{v}_{h}\right) \quad \forall\left\{\mathbf{v}_{h}, q_{1}\right\} \in V_{h} \times P_{1}
$$

where

$$
\begin{aligned}
B\left(\mathbf{w}_{h} ; \mathbf{u}_{h}, p_{1} ; \mathbf{v}_{h}, q_{1}\right)= & \left(\dot{\mathbf{u}}_{h}, \mathbf{v}_{h}\right)+\left(\left(\nabla \mathbf{u}_{h}\right) \mathbf{w}_{h}, \mathbf{v}_{h}\right)+\varepsilon\left(\nabla \mathbf{u}_{h}, \nabla \mathbf{v}_{h}\right) \\
& -\left(\nabla \cdot \mathbf{v}_{h}, p_{1}\right)+\left(\nabla \cdot \mathbf{u}_{h}, q_{1}\right)
\end{aligned}
$$

If we assume that the bubble part depends on space variables only, then the RFB method applied to the time-dependent problem leaves behind a stabilized finite element formulation of the SUPG type. With a temporal discretization of type (14), it reads: Find $\left\{\mathbf{u}_{1}^{s+1}, p_{1}^{s+1}\right\} \in V_{1} \times P_{1}$ such that

$$
\begin{aligned}
& \left(\mathbf{u}_{1}^{s+1}, \mathbf{v}_{1}\right)+\Delta t \alpha\left[\left(\left(\nabla \mathbf{u}_{1}^{s+1}\right) \mathbf{u}_{1}^{s+1}, \mathbf{v}_{h}\right)+\varepsilon\left(\nabla \mathbf{u}_{1}^{s+1}, \nabla \mathbf{v}_{1}\right)-\left(\nabla \cdot \mathbf{v}_{1}, p_{1}^{s+1}\right)-\left(\mathbf{f}, \mathbf{v}_{1}\right)\right] \\
& +\Delta t \alpha\left[\sum_{K \in T_{h}} \tau_{K} \int_{K}\left(\left[\dot{\mathbf{u}}_{1}^{s+1}+\left(\nabla \mathbf{u}_{1}^{s+1}\right) \mathbf{u}_{1}^{s+1}+\nabla p_{1}^{s+1}-\mathbf{f}\right] \cdot\left[\left(\nabla \mathbf{v}_{1}\right) \mathbf{u}_{1}^{s+1}-\nabla q_{1}\right]\right)\right] \\
& -\left(\mathbf{u}_{1}^{s}, \mathbf{v}_{1}\right)+\Delta t(1-\alpha)\left[\left(\left(\nabla \mathbf{u}_{1}^{s}\right) \mathbf{u}_{1}^{s}, \mathbf{v}_{1}\right)+\varepsilon\left(\nabla \mathbf{u}_{1}^{s}, \nabla \mathbf{v}_{1}\right)-\left(\nabla \cdot \mathbf{v}_{1}, p_{1}^{s}\right)-\left(\mathbf{f}, \mathbf{v}_{1}\right)\right] \\
& +\Delta t(1-\alpha)\left[\sum_{K \in T_{h}} \tau_{K} \int_{K}\left(\left[\dot{\mathbf{u}}_{1}^{s}+\left(\nabla \mathbf{u}_{1}^{s}\right) \mathbf{u}_{1}^{s}+\nabla p_{1}^{s}-\mathbf{f}\right] \cdot\left[\left(\nabla \mathbf{v}_{1}\right) \mathbf{u}_{1}^{s}-\nabla q_{1}\right]\right)\right] \\
& -\alpha\left(\nabla \cdot \mathbf{u}_{1}^{s+1}, q_{1}\right)-(1-\alpha)\left(\nabla \cdot \mathbf{u}_{1}^{s}, q_{1}\right)=0 \quad \forall\left\{\mathbf{v}_{1}, q_{1}\right\} \in V_{1} \times P_{1}
\end{aligned}
$$

where the values $\mathbf{u}_{1}^{s}$ and $p_{1}^{s}$ are known from the previous time step. The time derivatives in meshdependent terms are approximated by backward and forward difference operators, accordingly. The nonlinearity in the equation can be eliminated by a similar argument described in the previous section. 


\section{TWO-LEVEL ALGORITHM WITH A STABILIZING SUBGRID}

To compute the contribution of the RFB functions to the problem, we need to solve Equation (12) in each element, separately. Since Equation (12) can be viewed as a linear advection-diffusion equation, a cheap, yet efficient numerical algorithm can be employed to compute the approximate solution of the bubble problem as in [23, 24,26]: Specify a subgrid that consists of three vertices of the triangle plus a single additional node in the interior of each element and approximate the bubble function over the specified subgrid by choosing the location of the additional node such that it gives the best approximation in $L_{1}$ norm. These approximations will be used in problem (9) instead of the exact solutions of the bubble functions.

To describe the algorithm of the SSM, take a fixed element $K$ and consider a subgrid that contains just one additional node $N=N_{K}$ in the interior of each triangular element. We drop the subscript $K$ as the argument is similar for other elements. The node $N$ is joined to the three vertices denoted by $V_{i}$ splitting the triangle $K$ into three subtriangles. We will choose the point $N$ along one of the three medians of $K$. Let us denote the pseudo bubble basis function on $K$ by $b_{N}$ where it is defined as

$$
b_{N}(N)=1, \quad b_{N}\left(V_{i}\right)=0, \quad i=1,2,3
$$

Thus, the function $b_{N}$ attached to the point $N$ has support contained in $K$. We further denote the edge of $K$ opposite to $V_{i}$ by $\mathbf{e}_{i}$, the length of $\mathbf{e}_{i}$ by $\left|\mathbf{e}_{i}\right|$, the outward unit normal to $\mathbf{e}_{i}$ by $\mathbf{n}_{i}$ and $\boldsymbol{v}_{i}=\left|\mathbf{e}_{i}\right| \mathbf{n}_{i}$. The choice of the median on which the point $N$ is located will depend on the number of inflow edges in the triangle, and the precise location of $N$ will be chosen such that the value of the residual in the sense of $L_{1}$ is minimum. That is

$$
J(N)=\int_{K}\left|-\varepsilon \triangle b_{N}^{*}+\mathbf{u}_{1}^{n} \cdot \nabla b_{N}^{*}-1\right| \mathrm{d} K
$$

is minimum, where $b_{N}^{*}(\mathbf{x})=\alpha(N) b_{N}(\mathbf{x})$ is the unique solution of

$$
a_{K}\left(b_{N}^{*}, b_{N}\right)=\left(1, b_{N}\right) \quad \forall b_{N}
$$

Using the integration by parts, observe that

$$
\alpha(N)=\frac{\int_{K} b_{N} \mathrm{~d} K}{\varepsilon \int_{K}\left|\nabla b_{N}\right|^{2} \mathrm{~d} K}
$$

The set of points on the median $V_{1} M$ can be described as a function depending on a single parameter $t: N=(1-t) V_{1}+t M$, where $0<t<1$. In order to choose the position of $N$, we have to distinguish among two cases with respect to the number of inflow edges. Define the average velocity $\tilde{\mathbf{u}}_{1}^{n}$ by arithmetic mean of the velocity components at the vertices of the triangle; i.e. $\tilde{\mathbf{u}}_{1}^{n}=\left(\mathbf{u}_{1}^{n}\left(V_{1}\right)+\mathbf{u}_{1}^{n}\left(V_{2}\right)+\mathbf{u}_{1}^{n}\left(V_{3}\right)\right) / 3$. We use $\tilde{\mathbf{u}}_{1}^{n}$ to characterize the type of the edges of the triangle: If $\tilde{\mathbf{u}}_{1}^{n} \cdot \mathbf{n}_{i}>0$ then $\mathbf{e}_{i}$ is an outflow edge, otherwise it is an inflow edge. 

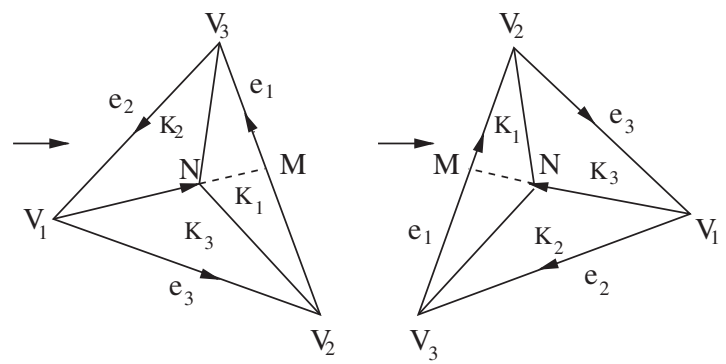

Figure 1. Types of inflow boundary: Case 1: Two inflow edges and Case 2: One inflow edge.

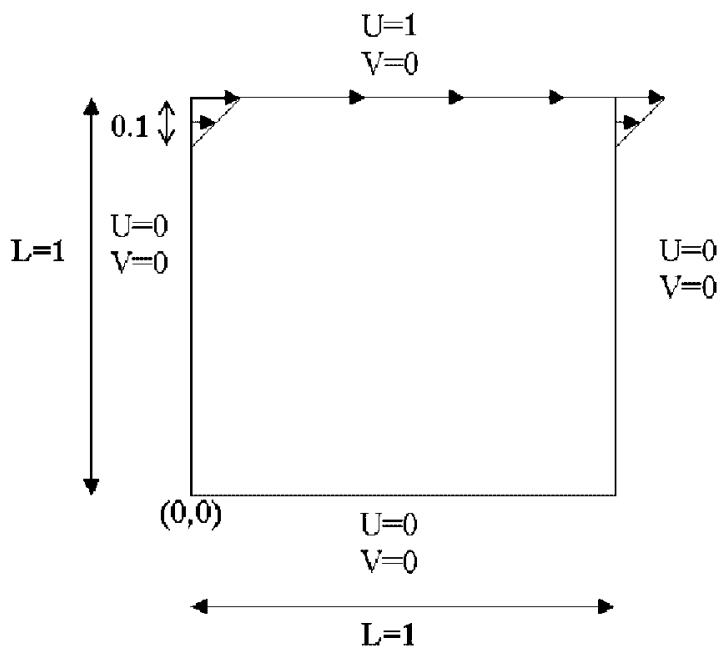

Figure 2. The problem statement of the lid-driven cavity flow.
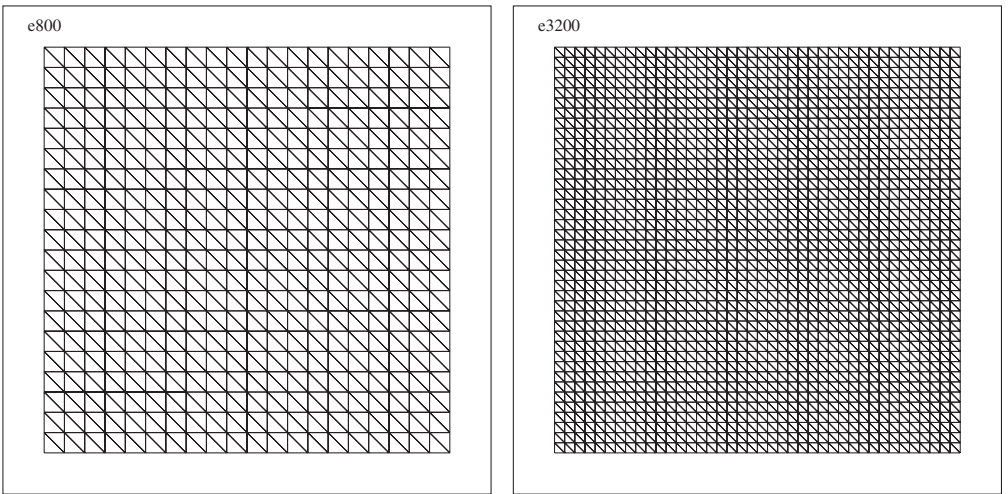

Figure 3. The problem meshes tested: 800 and 3200 elements. 

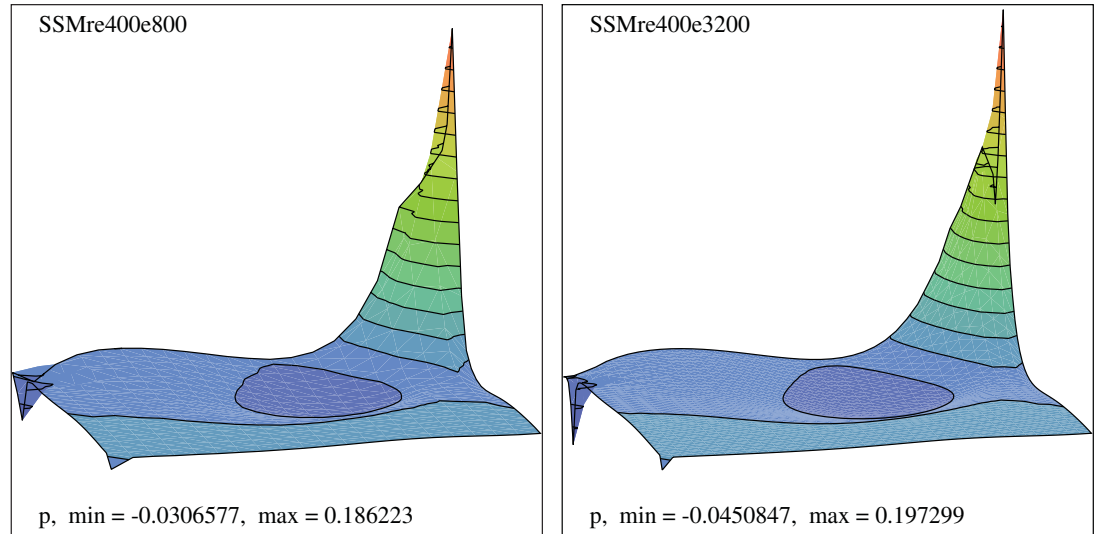

Figure 4. Pressure elevations for $R e=400$.
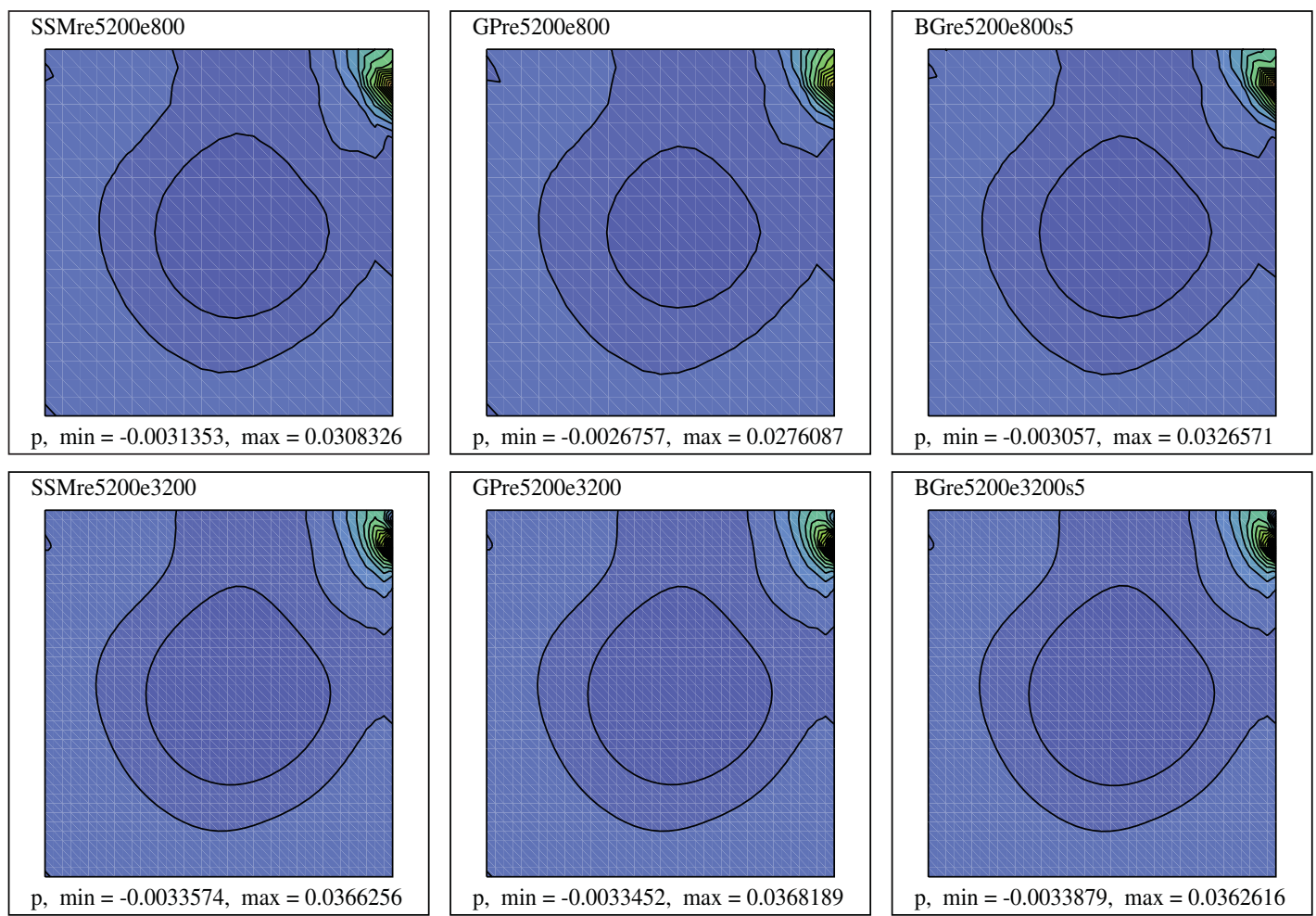

Figure 5. Pressure contours for $R e=5200$. 
In the first case where the inflow boundary is made up of two edges, let $\mathbf{e}_{2}$ and $\mathbf{e}_{3}$ be two inflow edges (Figure 1). Then for the value for $t$, we take

$$
\begin{array}{ll}
t=1+\frac{\varepsilon\left(\left|\mathbf{e}_{1}\right|^{2}\right)}{\varepsilon\left(\left|\mathbf{e}_{2}-\mathbf{e}_{3}\right|^{2}-\frac{2}{3}|K|\left(\tilde{\mathbf{u}}_{1}^{n}, \mathbf{v}_{1}\right)\right)} & \text { if } \varepsilon \leqslant \frac{2|K|\left(\tilde{\mathbf{u}}_{1}^{n}, \mathbf{v}_{1}\right) / 3}{3\left|\mathbf{e}_{1}\right|^{2}+\left|\mathbf{e}_{2}-\mathbf{e}_{3}\right|^{2}} \\
t=\frac{2}{3} & \text { otherwise }
\end{array}
$$

where $t$ lies in the interval $\left[\frac{2}{3}, 1\right)$. In the other case where the inflow boundary is made up of a single edge, let $\mathbf{e}_{1}$ be the inflow edge (Figure 1). We use again condition (18) to determine the location of $N$ and the integral $J(N)$ becomes minimum if $t$ is taken as

$$
\begin{array}{rlrl}
t & =\frac{\varepsilon\left(\left|\mathbf{e}_{2}\right|^{2}+\left|\mathbf{e}_{3}\right|^{2}\right)}{\varepsilon\left(\left|\mathbf{e}_{2}-\mathbf{e}_{3}\right|^{2} / 2-|K|\left(\tilde{\mathbf{u}}_{1}^{n}, \mathbf{v}_{1}\right) / 3\right)} & \text { if } \varepsilon \leqslant \frac{-2|K|\left(\tilde{\mathbf{u}}_{1}^{n}, \mathbf{v}_{1}\right) / 3}{3\left(\left|\mathbf{e}_{2}\right|^{2}+\left|\mathbf{e}_{3}\right|^{2}\right)-\left|\mathbf{e}_{2}-\mathbf{e}_{3}\right|^{2}} \\
t=\frac{2}{3} & \text { otherwise }
\end{array}
$$

In this case, $0<t \leqslant \frac{2}{3}$. We refer to [23, 26] for the derivation of the values of $t$ in (21) and (22). Two articles use two different criteria for choosing the subgrid node; however, they reach the similar results.
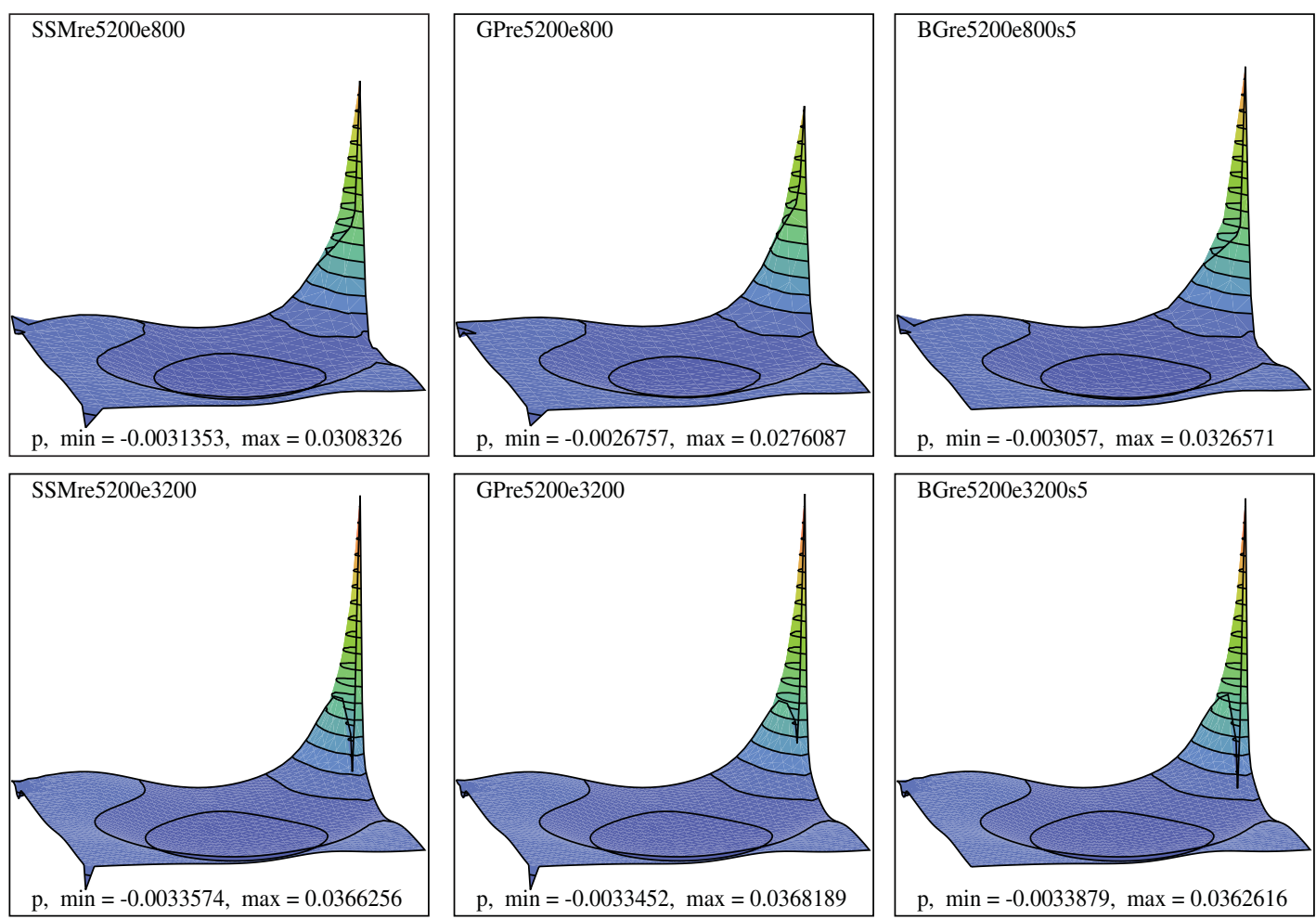

Figure 6. Pressure elevations for $R e=5200$. 

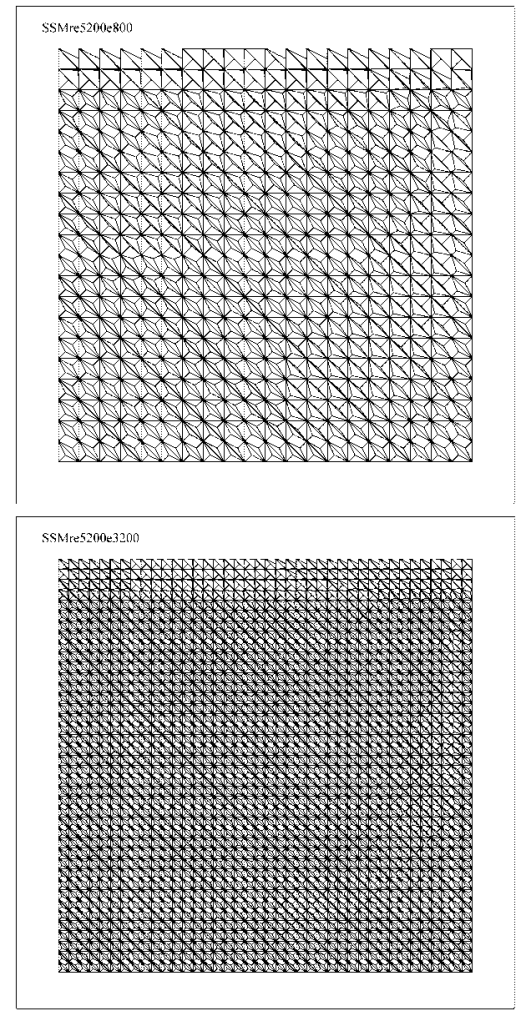

Figure 7. Adaptation of the subgrid points in SSM as the problem mesh is refined at $R e=5200$ : The problem meshes are $\mathbf{e} 800$ and $\mathbf{e} 3200$, respectively.
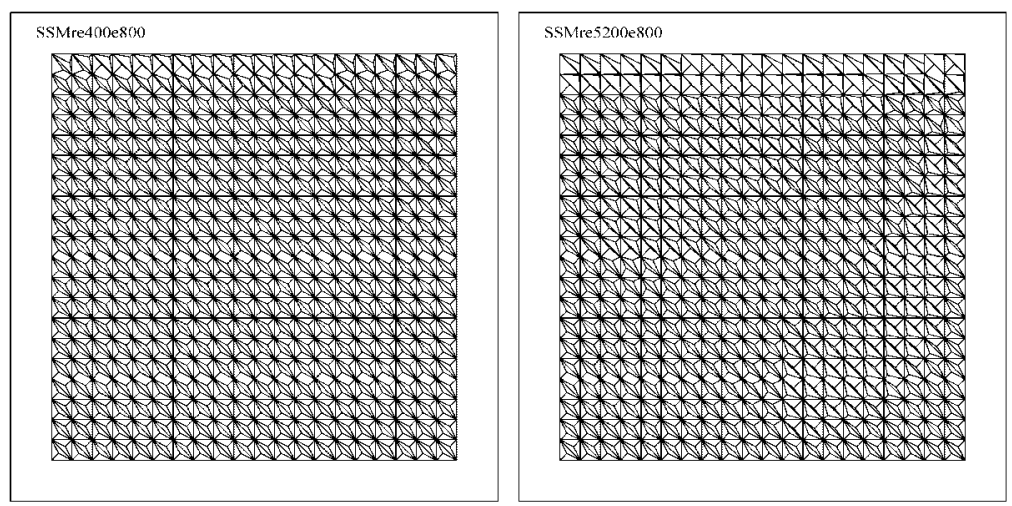

Figure 8. Adaptation of the subgrid points in SSM as the problem becomes advection dominated on a fixed mesh e800: $R e=400$ and 5200, respectively. 

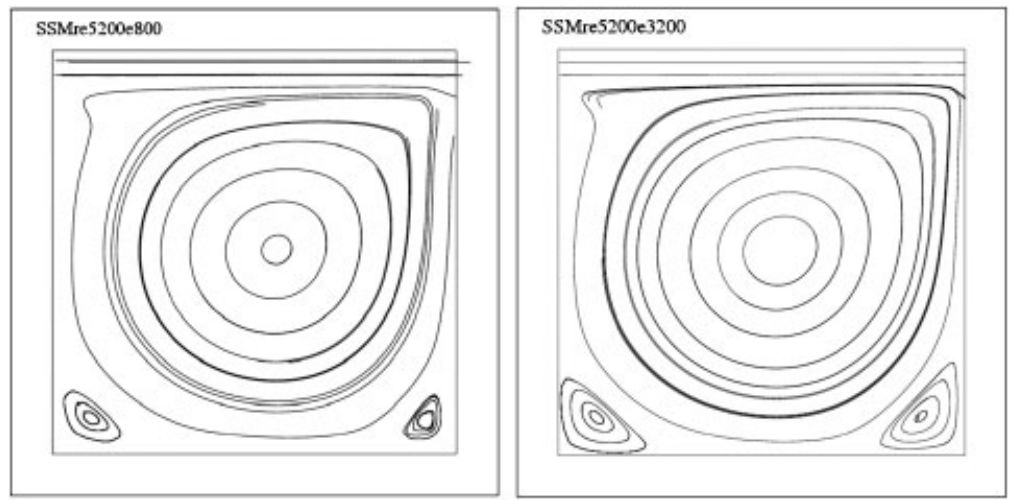

Figure 9. Streamlines for $R e=5200$.

Table I. Numerical values at some selected points through the geometric center of the cavity.

\begin{tabular}{lrrrrrr}
\hline \multicolumn{2}{l}{$u$ Velocity along vertical line $x=0.5$} & & \multicolumn{3}{c}{$v$ Velocity along horizontal line $y=0.5$} \\
\cline { 1 - 2 } \cline { 6 - 7 }$y$ & $R e=400$ & $R e=5200$ & & $x$ & $R e=400$ & $R e=5200$ \\
\hline 0.0000000 & 0.0000000 & 0.0000000 & & 0.0000000 & 0.0000000 & 0.0000000 \\
0.0750000 & -0.0350700 & -0.0648715 & & 0.0750000 & 0.0530557 & 0.0506292 \\
0.1250000 & -0.0548183 & -0.0819039 & & 0.1250000 & 0.0734340 & 0.0669750 \\
0.2500000 & -0.0977078 & -0.0588283 & & 0.2500000 & 0.0969054 & 0.0650425 \\
0.3750000 & -0.1107317 & -0.0293358 & & 0.3750000 & 0.0939595 & 0.0359474 \\
0.5000000 & -0.0645859 & -0.0002669 & & 0.5000000 & 0.0571612 & 0.0097147 \\
0.6250000 & 0.0137531 & 0.0308169 & & 0.6250000 & -0.0158838 & -0.0171241 \\
0.7500000 & 0.0858983 & 0.0664360 & & 0.7500000 & -0.1208616 & -0.0467028 \\
0.8500000 & 0.1736514 & 0.0834718 & & 0.8500000 & -0.1819628 & -0.0721858 \\
0.9000000 & 0.3088252 & 0.1397576 & & 0.9000000 & -0.1584394 & -0.1042040 \\
0.9500000 & 0.5886718 & 0.5006183 & & 0.9500000 & -0.0880898 & -0.1083465 \\
0.9750000 & 0.7852072 & 0.7521335 & & 0.9750000 & -0.0438278 & -0.0614507 \\
1.0000000 & 1.0000000 & 1.0000000 & & 1.0000000 & 0.0000000 & 0.0000000 \\
\hline
\end{tabular}

Once the location of the point $N$ is determined through the relation $N=(1-t) V_{1}+t M$ with the value of $t$ given in (21) or (22) depending on the number of inflow edges, a reasonably good approximation to the stabilization parameter $\tau_{K}$ can be obtained: The approximate value of $\tau_{K}$ is given by

$$
\tilde{\tau}_{K}=\frac{1}{|K|} \int_{K} b_{N}^{*}=\frac{1}{|K|} \frac{\left(\int_{K} b_{N}\right)^{2}}{\varepsilon \int_{K}\left|\nabla b_{N}\right|^{2}}=\frac{4|K|}{9 \varepsilon \sum_{i}\left|e_{i}\right|^{2} /\left|K_{i}\right|}
$$

where $K_{i}$ is the area of $i$ th subtriangle. The values of $\tilde{\tau}_{K} \mathrm{~s}$ are then used in the global formulation (9) in place of $\tau_{K}$. A numerical approximation consistent with the physical configuration of the problem can now be obtained for the piecewise-linear part of the solution through (9). 


\section{NUMERICAL RESULTS}

In this section, we present some numerical results with the SSM presented above and compare it with the methods employing some other variants of stabilizing subgrids. We work on three test problems: (1) 2D laminar flow inside a lid-driven cavity, (2) 2D flow past a cylinder, (3) an unsteady flow around a cylinder. We use the tool VIGIE (Visualization Generale Interactive d'Ecoulements) to visualize the numerical results. The iteration cycle that resolves the nonlinearity of the problem stops when the maximum norm of the error is less than $10^{-6}$.

Before we present numerical results we briefly mention our convention in labels. The upper case letters at the beginning of each label refers to the method we employ at the subgrid level: SSM
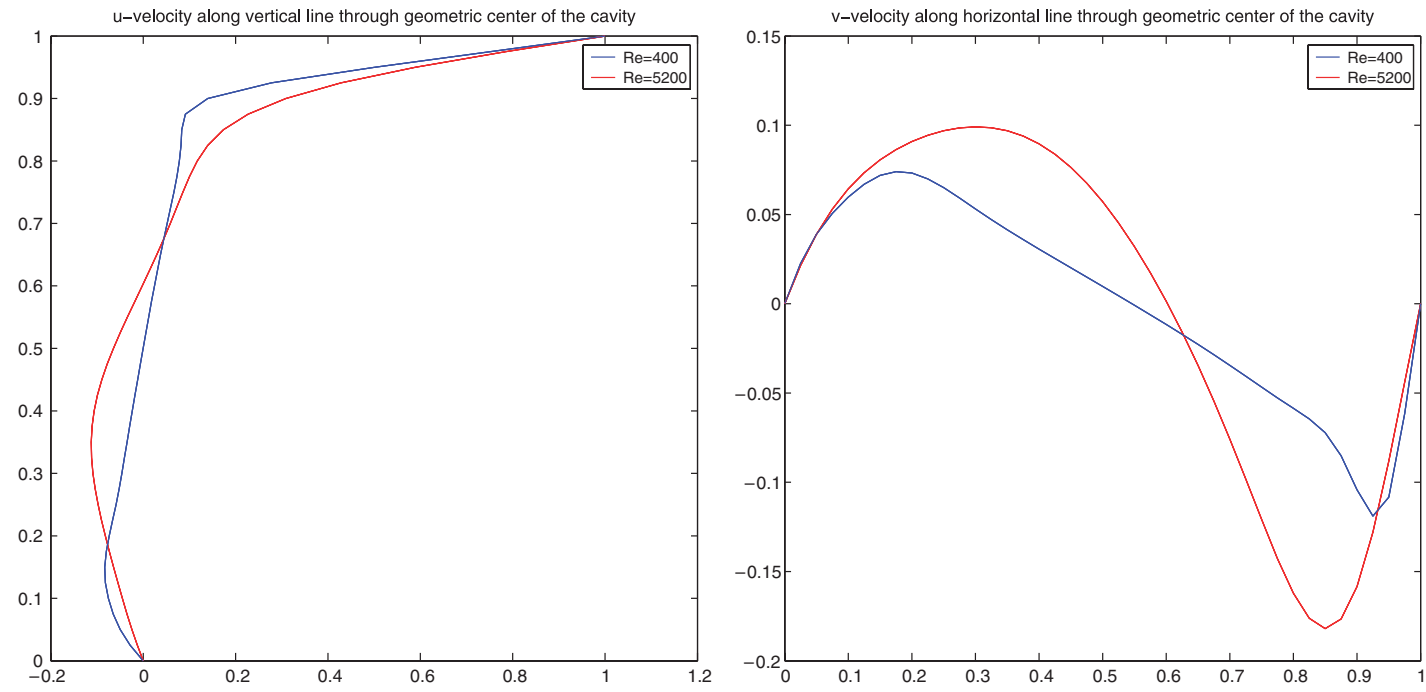

Figure 10. Velocity profiles for $R e=400$ and 5200 .

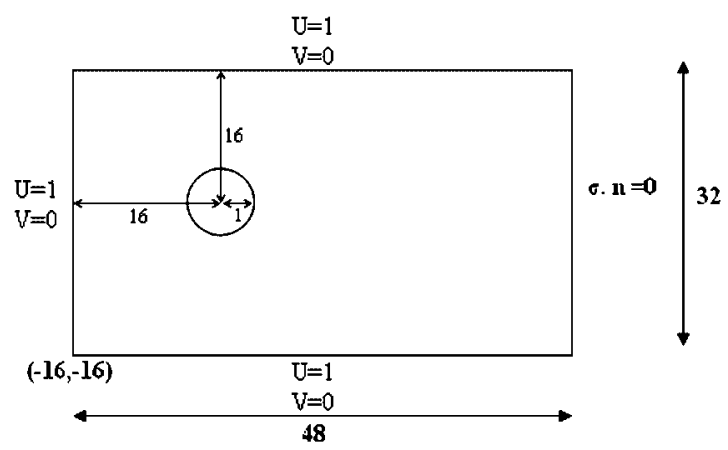

Figure 11. The statement of the flow problem past a cylinder. 
refers to the stabilizing subgrid method, $G P$ refers to the method selecting subgrid point at the gravity center of each element and $B G$ refers to the TLFEM in which the subgrid is the finest and generated by repeating the subgrid strategy in $G P$ to subtriangles $\mathbf{s}$ times. Numerical experiments show that the optimum value for $\mathbf{s}$ is 5 . Hence, we keep the value of $\mathbf{s}$ fixed and take $\mathbf{s}=5$ throughout the calculations. What follows the part of the label referring to the method employed is the Reynolds number tag that we denote it by re. The number of elements on the global mesh follows e. Thus, for example, the label SSMre26e6800 means that, for the problem of interest, we test the SSM at Reynolds number 26 over a global mesh with 6800 elements. We remark that all three methods use the same formulation at the global scale.
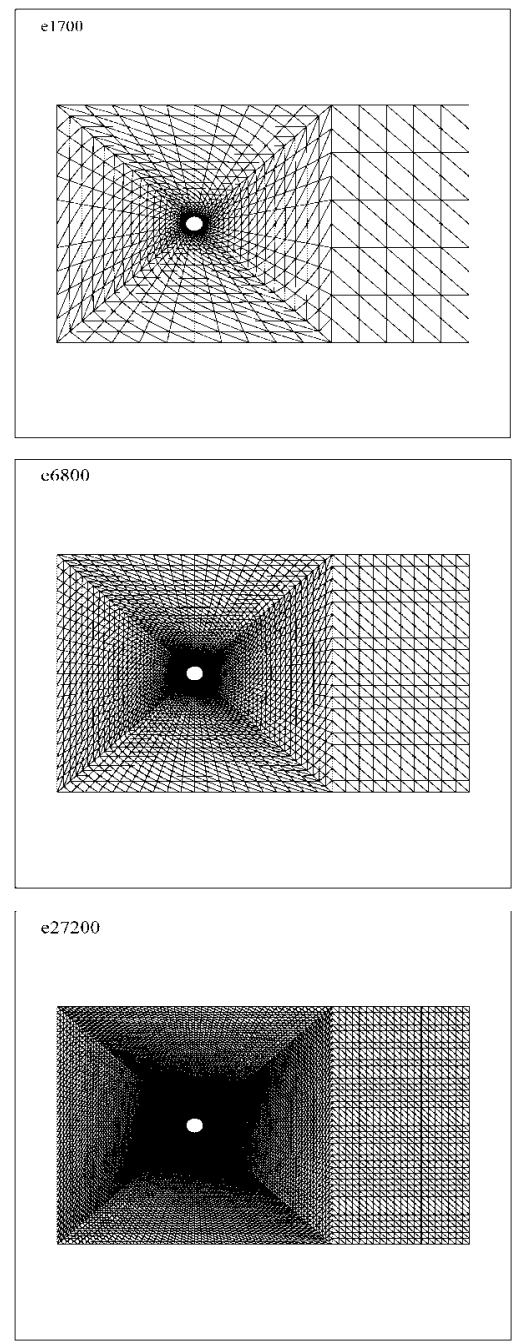

Figure 12. The problem meshes tested. 


\subsection{Cavity flow}

This is a standard benchmark problem. The problem specifications are given in Figure 2. We impose continuous boundary conditions by means of a linear transition from the level $y=0.9$ to 1.0 on the upper corners of the boundary, and this behavior remains unchanged with mesh refinement. The Reynolds number is based on the characteristic velocity $U$ and the characteristic length $L$ and is given by

$$
\operatorname{Re}=\frac{U L}{\varepsilon}
$$

Since the pressure is determined up to a constant through the formulation, we append the condition

$$
\int_{\Omega} p=0
$$

to the formulation to fix the constant. For problems having high Reynolds number, the convergence of the iteration is only attained under a good initial guess. To provide such a guess, we use a continuation process on the top of the iteration.
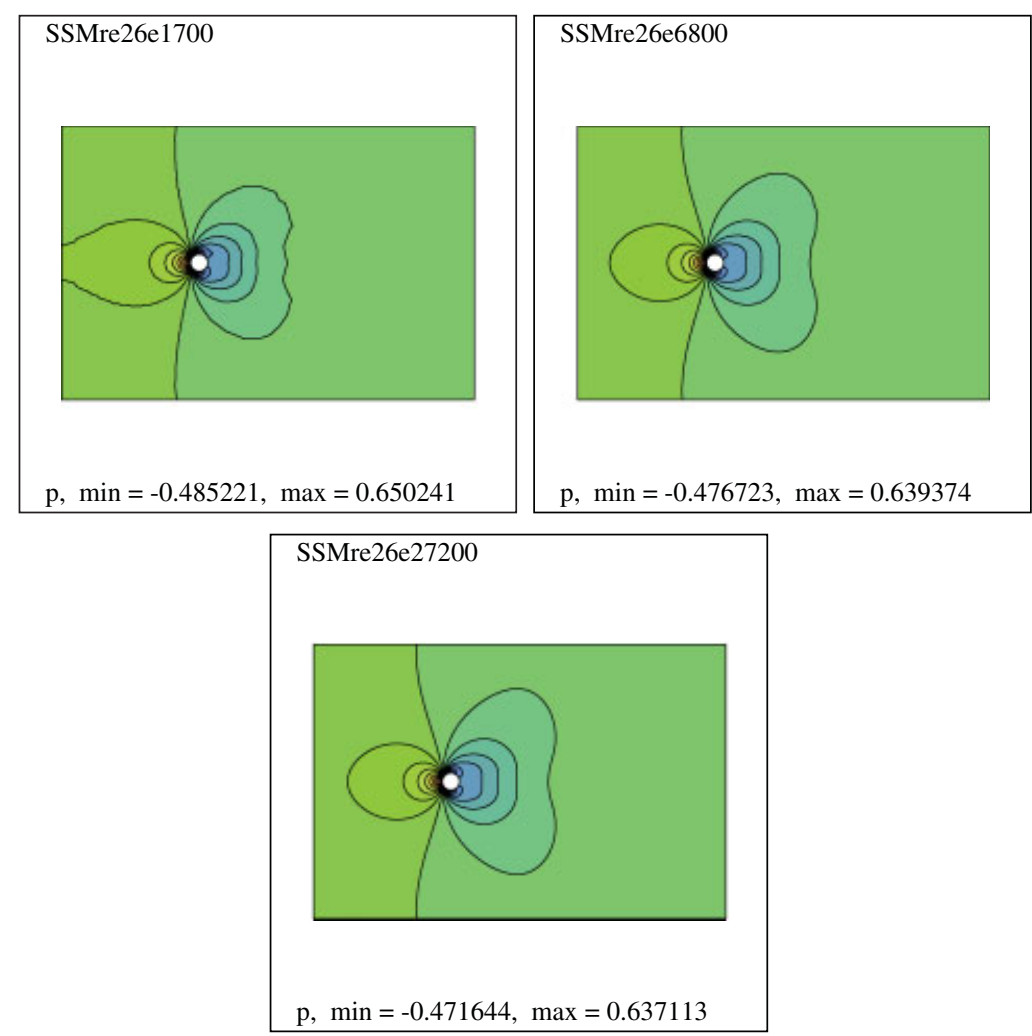

Figure 13. The cylinder problem: Pressure contours for $R e=26$. 

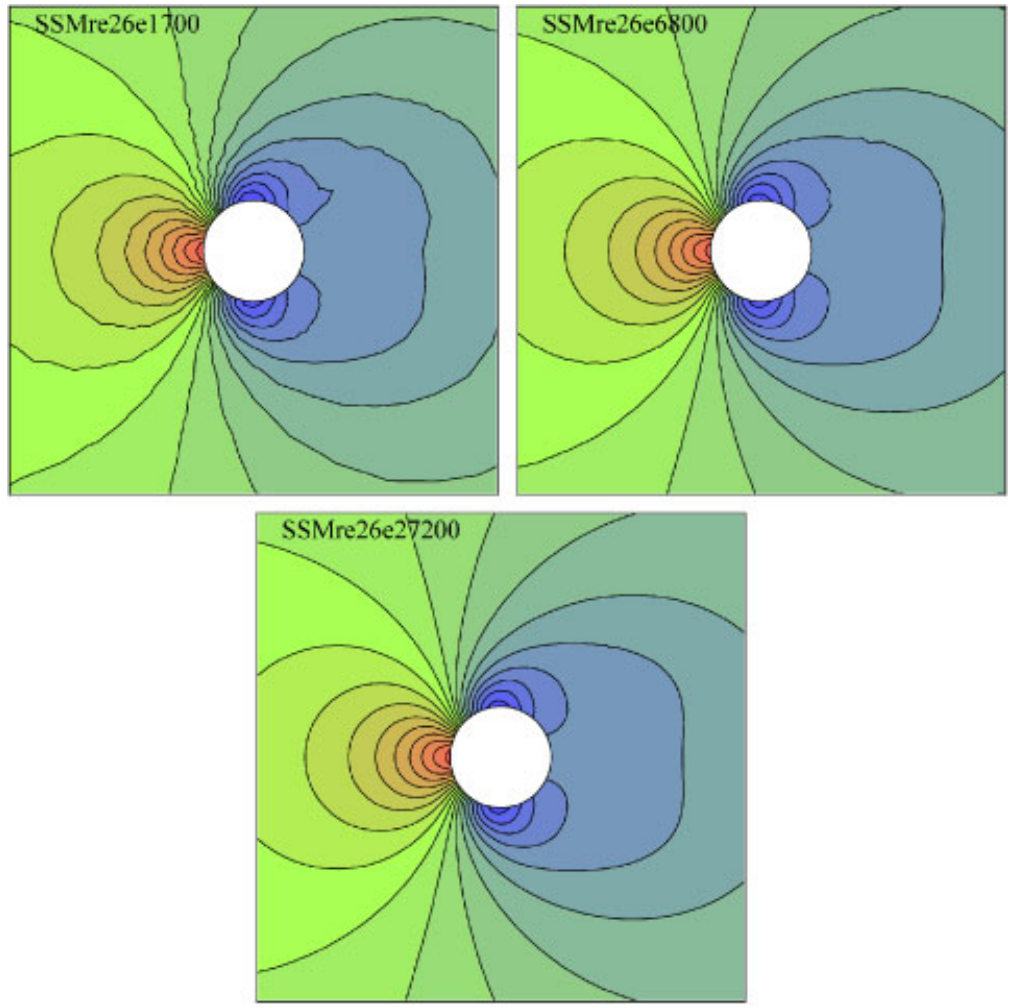

Figure 14. Pressure detail behind the cylinder for $R e=26$.
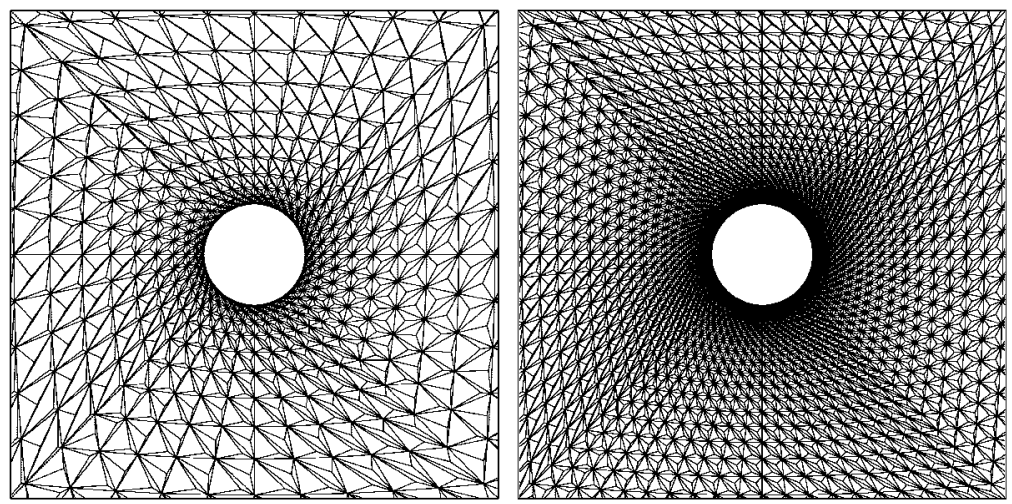

Figure 15. Mesh detail around the cylinder for $R e=26$ with $\mathbf{e}=1700$ and 6800 .

We present the numerical results for $R e=400$ and 5200 on a pair of successively refined uniform meshes (see Figure 3). For $R e=400$, the flow regime of the real solution is diffusive dominated 

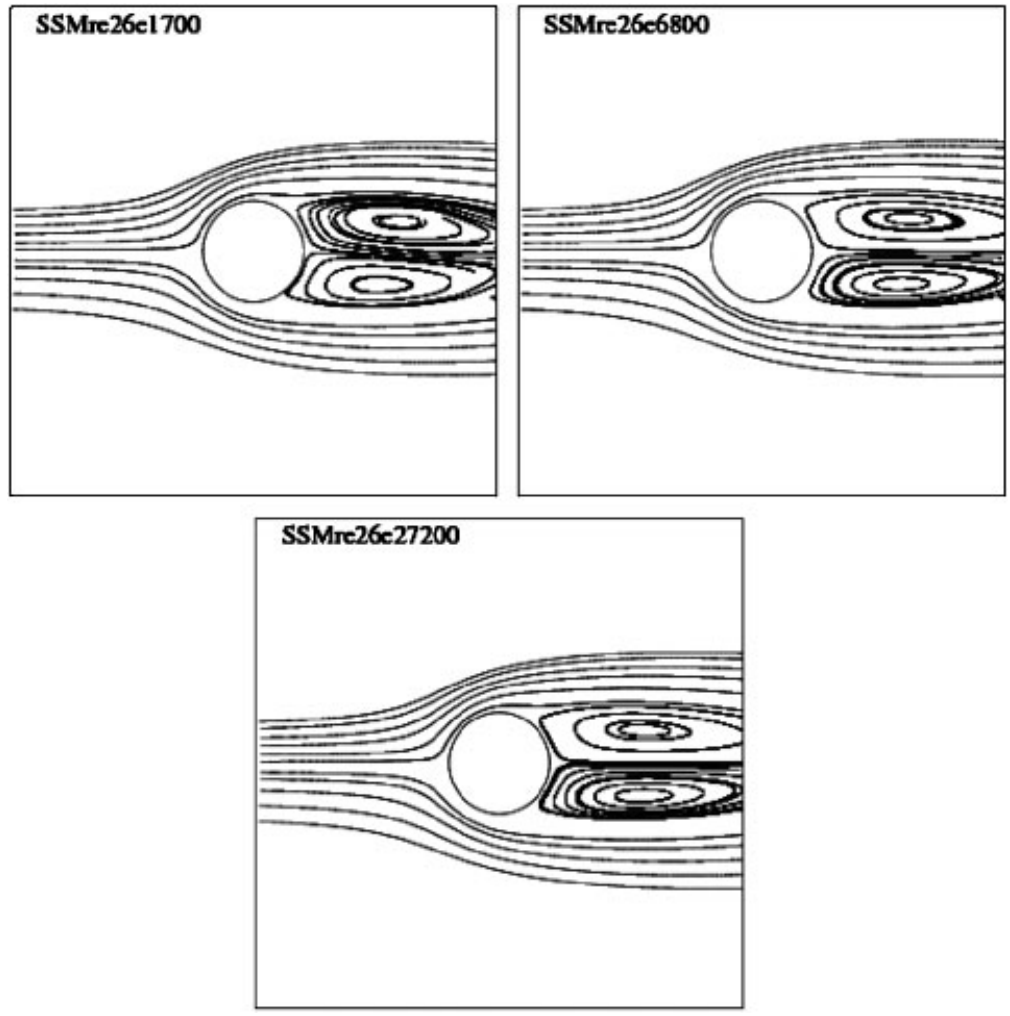

Figure 16. Streamlines detail behind the cylinder for $R e=26$.

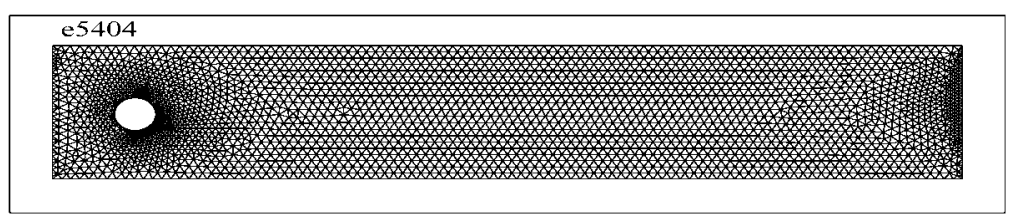

Figure 17. The unsteady flow around a cylinder problem, mesh tested: 5404 triangular elements.

in most parts of the domain, and the roles of stabilization components are not so significant. Therefore, all three methods produce similar results and we present the pressure elevations of the numerical approximations obtained through SSM only in Figure 4. They show the characteristic features of the real solution that are comparable with the results present in the literature [22, 27]. Figures 5 and 6 show pressure contours and pressure elevations, respectively, at Reynolds number $R e=5200$. Assuming that the results obtained through the TLFEM are the most accurate, we may conclude that SSM predicts the real solution better than GP at both levels of the problem meshes. This shows that the proper choice of the subgrid node may play a significant role in obtaining more accurate approximations. As the mesh gets finer, the problem becomes locally diffusive dominated 

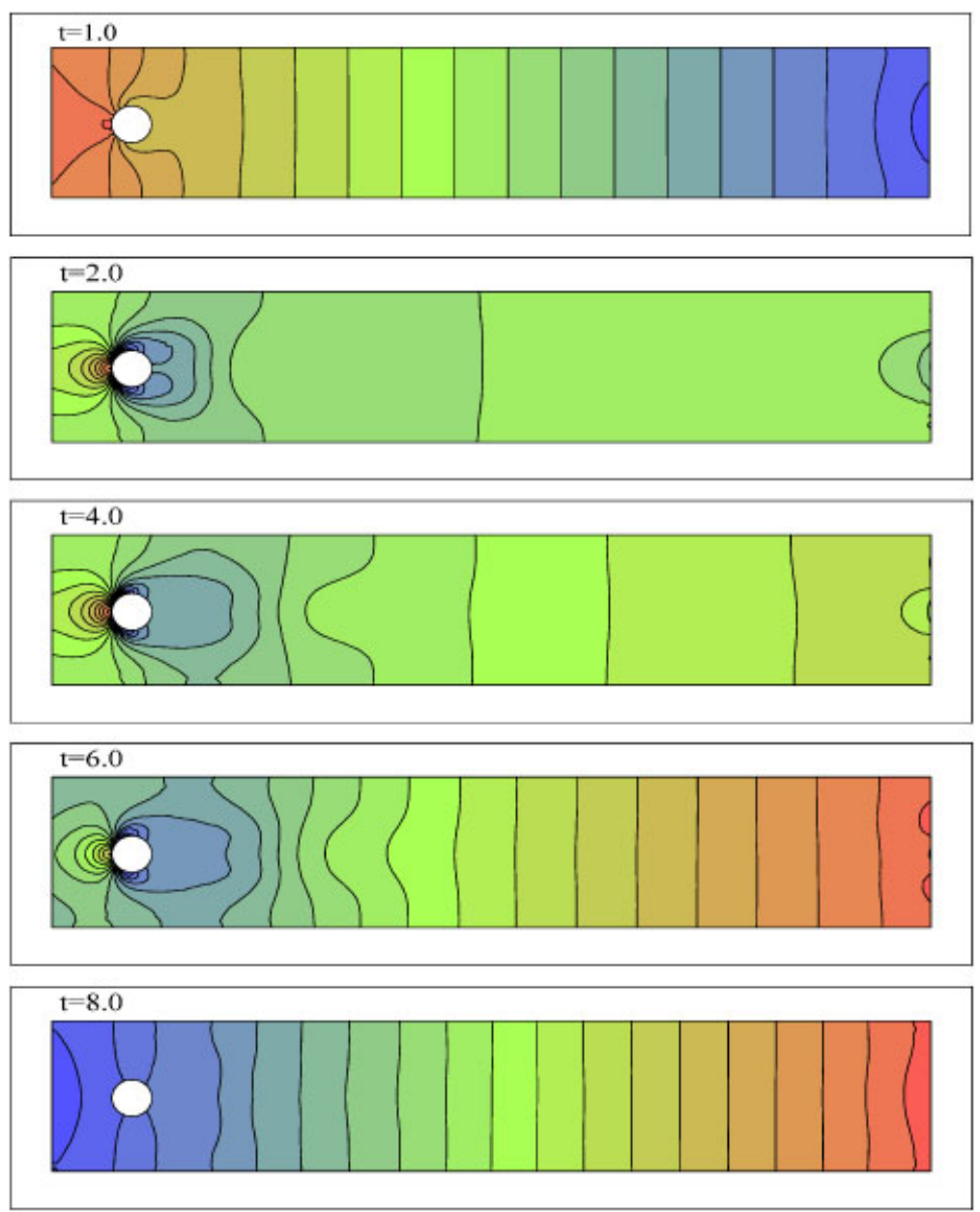

Figure 18. Pressure contours for unsteady flow around a cylinder problem at $t=1,2,4,6$ and 8 , respectively.

in a larger portion of the domain and the need for stabilization is diminished (Figure 7). The situation is apparent as the location of the subgrid points suggested by SSM moves to the gravity center of the element and all three solutions get closer to each other. However, SSM is slightly better that GP as SSM suggests a more correct location for the subgrid node over a smaller portion of the domain (Figure 7).

On the other hand, Figures 8 shows the configuration of subgrid points in the mesh e 800 for $R e=400$ and 5200, respectively. As the Reynolds number increases, the problem becomes advection dominated and therefore the adaptation of the position of the subgrid point is strongly pronounced. The stabilization is now effective on a larger portion of the entire domain. Streamlines presented in Figure 9 for $R e=5200$ with the $S S M$ are also in agreement with the results in the literature (see $[22,28,29])$. As given in Reference [30], the approximate results of velocity components through the geometric center of the cavity are presented in Table I and Figure 10, respectively. 

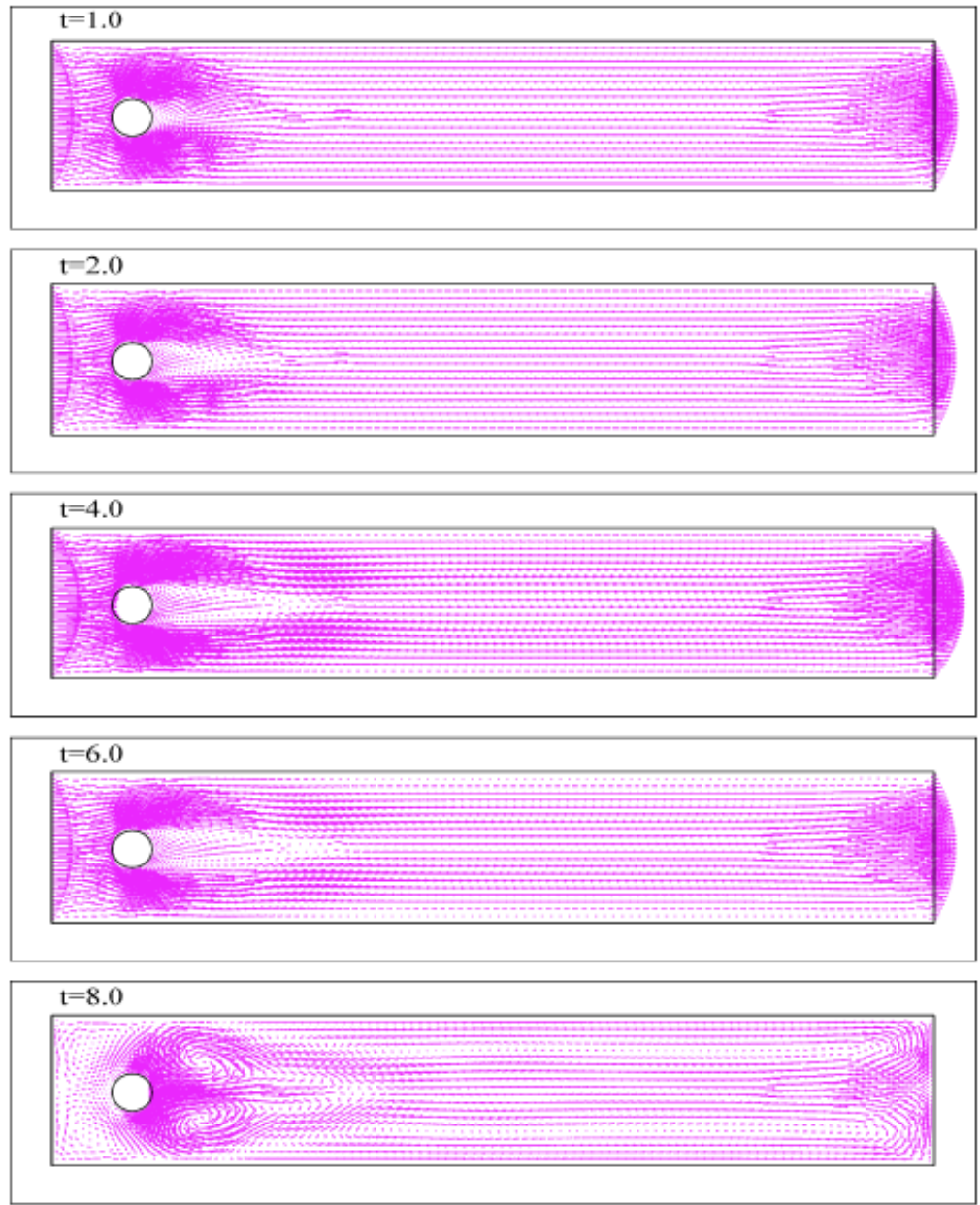

Figure 19. Flow vectors for unsteady flow around a cylinder problem at $t=1,2,4,6$ and 8 , respectively.

\subsection{Flow past a cylinder}

The geometry and the boundary conditions are shown in Figure 11. At upper and lower computational boundaries and at the inflow section, a uniform free-stream velocity boundary condition is imposed. The traction-free condition is imposed at the outflow boundary. The steady flow past a cylinder is studied at $R e=26$, where the Reynolds number is based on the free-stream velocity and the cylinder diameter, i.e. $R e=\left(U_{\infty} d\right) / \varepsilon$. See [31] for a historical overview of this problem.

We are interested in the performance of the SSM over a series of successively refined meshes (Figure 12). Around the cylinder, we employ a uniform distribution of 40,80,160 nodes in angular direction and a quadratic distribution of $21,41,81$ nodes in radial direction, respectively. We present the pressure contours for $R e=26$ in Figure 13. Although the method captures the main features of the exact solution, even at the very coarse level of the global mesh, the increase in the number 
of elements improves the approximation. A detailed plot of pressure contours around the cylinder in Figure 14 confirms this observation.

We note that the SSM is more effective over coarser meshes. This can be observed in Figure 15, as SSM adapts the location of the subgrid node over a larger portion of the region. This adaptation is crucial in obtaining stabilized approximations. We note that the streamlines in Figure 16 are in good agreement with the experimental results in [32].

\subsection{Unsteady flow around a cylinder}

The unsteady flow around a cylinder problem [33] is governed by the unsteady Navier-Stokes equations with $\operatorname{Re}=100$ and $\mathbf{f}=0$. The problem is given with homogeneous initial value $\mathbf{u}(x, y, 0)=$ 0 and boundary conditions at inflow $(x=0)$ and outflow $(x=2.2)$ boundaries as

$$
\mathbf{u}(0, y, t)=\mathbf{u}(2.2, y, t)=0.41^{-2} \sin (\pi t / 8)(6 y(0.41-y), 0), \quad 0 \leqslant y \leqslant 0.41
$$

No-slip boundary conditions are described at the other boundaries $(y=0,0.41$ and around the cylinder). It is seen from the given boundary conditions that the problem has periodic behavior at $T=8$. Therefore, the problem is solved in the time domain for $t \in[0,8]$ using Crank-Nicolson scheme with a time step $\Delta t=0.1$. In space dimension, the problem domain is discretized by 5404 triangular linear elements (Figure 17). The efficiency of the applied numerical procedure is observed from the obtained results in terms of figures. The periodic behavior of the flow is displayed as pressure contours in Figure 18. Also, Figure 19 shows the unsteady behavior of the flow in terms of flow vectors. Although the large time step is used in the solution procedure, characteristic of the flow is captured by SSM.

\section{CONCLUSION}

We consider an SSM for the approximate solution of the incompressible Navier-Stokes equations in the framework of the TLFEM. The domain is planar and the discretization is triangular. The presentation above shows that the TLFEM with the stabilizing subgrid produces stable and accurate approximations in a variety of problem configurations establishing that it is applicable to nonlinear problems in 2 dimensions. Numerical experiments further indicate that the proper choice of the subgrid node may play a significant role in obtaining more accurate approximations, especially for high Reynolds number.

\section{ACKNOWLEDGEMENTS}

This work is supported by The Scientific and Technical Research Council of Turkey (TUBITAK) (Contract \# 105T091).

\section{REFERENCES}

1. Oden JT, Wellford LC. Analysis of flow of viscous fluids by the finite element method. AIAA Journal 1972; 10:1590-1599.

2. Taylor C, Hood P. A numerical solution of the Navier-Stokes equations using the finite element technique. Computers and Fluids 1973; 1:73-100. 
3. Brezzi F. On the existence, uniqueness and approximation of saddle-point problems arising from Lagrange multipliers. RAIRO Série Rouge 1974; 8:129-151.

4. Babuska I. The finite element method with Lagrangian multipliers. Numerische Mathematik 1973; 20:179-192.

5. Roos HG, Stynes M, Tobiska L. Numerical Methods for Singularly Perturbed Differential Equations. Springer: Berlin, 1996.

6. Brooks AN, Hughes TJR. Streamline upwind/Petrov-Galerkin formulations for convection dominated flows with particular emphasis on the incompressible Navier-Stokes equations. Computer Methods in Applied Mechanics and Engineering 1982; 32:199-259.

7. Johnson C, Nävert U, Pitkäranta J. Finite element methods for linear hyperbolic problem. Computer Methods in Applied Mechanics and Engineering 1984; 45:285-312.

8. Brezzi F, Bristeau MO, Franca LP, Mallet M, Rogé G. A relationship between stabilized finite element methods and the Galerkin method with bubble functions. Computer Methods in Applied Mechanics and Engineering 1992; 96:117-129.

9. Brezzi F, Russo A. Choosing bubbles for advection-diffusion problems. Mathematical Models and Methods in Applied Sciences 1994; 4:571-587.

10. Hughes TJR. Multiscale phenomena: Green's functions, the Dirichlet-to-Neumann formulation, subgrid scale models, bubbles and the origin of stabilized methods. Computer Methods in Applied Mechanics and Engineering 1995; 127:387-401.

11. Franca LP, Farhat C. Bubble functions prompt unusual stabilized finite element methods. Computer Methods in Applied Mechanics and Engineering 1995; 123:299-308.

12. Russo A. Bubble stabilization of finite element methods for the linearized incompressible Navier-Stokes equations. Computer Methods in Applied Mechanics and Engineering 1996; 132:335-343.

13. Brezzi F, Franca LP, Hughes TJR, Russo A. Stabilization techniques and subgrid scales capturing. The Proceedings of the Conference 'State of the Art in Numerical Analysis', York, England, April 1-4, 1996.

14. Brezzi F, Franca LP, Hughes TJR, Russo A. $b=\int g$. Computer Methods in Applied Mechanics and Engineering 1997; 145:329-339.

15. Baiocchi C, Brezzi F, Franca LP. Virtual bubbles and the Galerkin-least-squares method. Computer Methods in Applied Mechanics and Engineering 1993; 105:125-141.

16. Franca LP, Nesliturk A, Stynes M. On the stability of residual-free bubbles for convection-diffusion problems and their approximation by a two-level finite element method. Computer Methods in Applied Mechanics and Engineering 1998; 166:35-49.

17. Brezzi F, Franca LP, Russo A. Further considerations on residual-free bubbles for advective-diffusive equations. Computer Methods in Applied Mechanics and Engineering 1998; 166:25-33.

18. Brezzi F, Hughes TJR, Marini LD, Russo A, Suli E. A priori error analysis of residual-free bubbles for advection-diffusion problems. SIAM Journal on Numerical Analysis 1999; 36:1933-1948.

19. Brezzi F, Marini LD, Suli E. The residual-free bubbles for advection-diffusion problems: the general error analysis. Numerische Mathematik 2000; 85:31-47.

20. Sangalli G. Global and local error analysis for the residual-free bubbles method applied to advection-dominated problems. SIAM Journal on Numerical Analysis 2000; 38:1496-1522.

21. Franca LP, Macedo AP. A two-level finite element method and its application to the Helmholtz equation. International Journal for Numerical Methods in Engineering 1998; 43:23-32.

22. Nesliturk A. Approximating the incompressible Navier-Stokes equations using a two level finite element method. Ph.D. Thesis, University of Colorado, Denver, 1999.

23. Brezzi F, Marini D, Russo A. Applications of the pseudo residual-free bubbles to the stabilization of convectiondiffusion problems. Computer Methods in Applied Mechanics and Engineering 1998; 166:51-63.

24. Brezzi F, Marini D, Russo A. On the choice of a stabilizing subgrid for convection-diffusion problems. Computer Methods in Applied Mechanics and Engineering 2005; 194:127-148.

25. Ciarlet PG. The Finite Element Methods for Elliptic Problems. North-Holland: Amsterdam, 1978.

26. Nesliturk AI. A stabilizing subgrid for convection-diffusion problem. Mathematical Models and Methods in Applied Sciences 2006; 16(2):211-231.

27. Tezduyar TE, Shih R, Mittal S, Ray SE. Incompressible flow computations with stabilized bilinear and linear equal-order-interpolation velocity-pressure elements. Computer Methods in Applied Mechanics and Engineering 1992; 95:221-242.

28. Sohn JL. Evaluation of FIDAP on some classical laminar and turbulent benchmarks. International Journal for Numerical Methods in Fluids 1988; 8:1469-1490. 
29. Liou J, Tezduyar T. Clustered element-by-element computations for fluid flow. In Parallel Computational Fluid Dynamics: Implementations and Results, Simon HD (ed.). MIT Press: Cambridge, MA, 1990; 165-188.

30. Ghia U, Ghia KN, Shin CT. High-resolutions for incompressible flow using the Navier-Stokes equations and a multigrid method. Journal of Computational Physics 1982; 48:387-411.

31. Fornberg B. Computing steady incompressible flows past blunt bodies: a historical overview. In Numerical Methods for Fluid Dynamics, vol. 4, Baines MJ, Morton KW (eds). Clarendon Press: London, 1993; 115-133.

32. Van Dyke M. An Album of Fluid Motion. The Parabolic Press: California, 1982.

33. John V. Reference values for drag and lift of a two dimensional time-dependent flow around a cylinder. International Journal for Numerical Methods in Fluids 2004; 44:777-788. 